\title{
Embedded Control using Mems Sensor with Voice Command and CCTV Camera
}

\author{
E. Kanniga ${ }^{1 *}$, K. Selvaramarathnam ${ }^{2}$ and M. Sundararajan ${ }^{3}$ \\ 'Research Scholar, Department of Electronics and Communication, Bharath University, Chennai-73; \\ kannigatruth@yahoo.com \\ ${ }^{2}$ PG Scholar, Technology of Applied Electronics, Bharath University, Tamil Nadu,Chennai-73; \\ iasrathnam1@gmail.com \\ 3Principal, Sri Lakshmiammal Engineering College, Chennai-600 073; \\ msrajan69@gmail.com
}

\begin{abstract}
The aim of our study aims to design a wheelchair having network controlled by memes sensor with voice command and CCTV camera. This work is intended to help and blind persons. Our work enables a dumb person sitting on wheel chair can press a keypad to generate sound from voice board.
\end{abstract}

Keyword: MEMS, IRsensor, Circuit Relay

\section{Introduction}

\subsection{Hardware Description}

The hardware is based on AT89S52 microcontroller, which is compatible with 51 family microcontrollers. The AT89S52 is a low power, high performance CMOS 8-bit microcontroller with $8 \mathrm{k}$ bytes of in-system programmable Flash memory. MEMS sensor is used to give the direction, accordingly the wheel chair moves by using relay and motors. Power supply is given to the microcontroller. IR sensors are used to detect the obstacles and automatically turn off the wheel chair.

\subsection{Software Description}

The basic software for microcontroller is embedded C language. It is compiled using KEIL compiler. The compiler produces the final machine code of the program and it is stored in the flash memory of the microcontroller.

The objective of this project was to design a wheelchair which is controlled by MEMS sensor with voice command and CCTV camera. This work is intended to help patients and blind persons. Person sitting on wheel chair who may be a dumb can press a keypad generate sound that comes from the voice board.

The Embedded software code used to monitor wheel chair or movement that are not typically thought of as machine. This plays an integral role in the electronic machine it is supplied with specific hardware. Reading data sheets for discrete components to determine usage of registers and camera system. Power supply is a reference to a source of electrical power. A device or system that supplies electrical or other types of energy to an output load or group of loads is called a power supply unit or PSU as per Figure 1.

This is possible because it has completed range of products to suit every situation and any industry and also using the multi national presence. With emerging needs of surveillance and monitoring solution today, CCTVTECH seeks out technology from all corners of the world and form a strategic alliance with technologydriven manufacturing firms of different product categories in CCTV such as the Fiber Optical Transceiver devices, Twisted Pair Transceiver devices, Digital Recording 
systems, and IP solutions to integrate with its professional expertise [5].

The technical tenure is most commonly applied to electrical energy supplies, less often to automatic ones, and rarely to others.

A $230 \mathrm{v}, 50 \mathrm{~Hz}$ Single phase AC power supply is set to a step down transformer to get a hold $12 \mathrm{v}$ supply. This applied voltage is converted to DC voltage using a power pack circuit. The converted pulsating line voltage is controlled by a $2200 \mathrm{uf}$ capacitor and then given to 7805 voltage regulator to gain constant $5 \mathrm{v}$ supply. This $5 \mathrm{v}$ supply is given to all the components in the circuit. A RC time constant circuit is added to discharge all the capacitors quickly. To ensure the power supply a light emitting diode is connected for indication purpose.

Microcontroller receives $5 \mathrm{v}$ from 7805 voltage regulator. It has four ports namely port 0 , port 1 , port 2 and port 3 . Relay is an electrical switch which is connected to port 2 of a microcontroller. MEMS sensor is an acceleration sensor which senses acceleration. It is used to give the direction of wheel chair. IR sensor is also connected to the microcontroller. It is used to detect obstacle. It is having two parts one is transmitter and other part is receiver. Transmitter sends infra red to objects and reflected infra red from object is received by receiver. In this way IR sensor senses obstacle.

The board of Voice is connected to microcontroller. It is having APR9600 multi section sound recorder/replay IC. APR9600 is a low-cost high performance sound record/ replay IC incorporating flash analogue storage technique. Recorded sound is retained even after power supply is removed from the module. The replayed sound exhibits high quality with a low noise level. Sampling rate for a

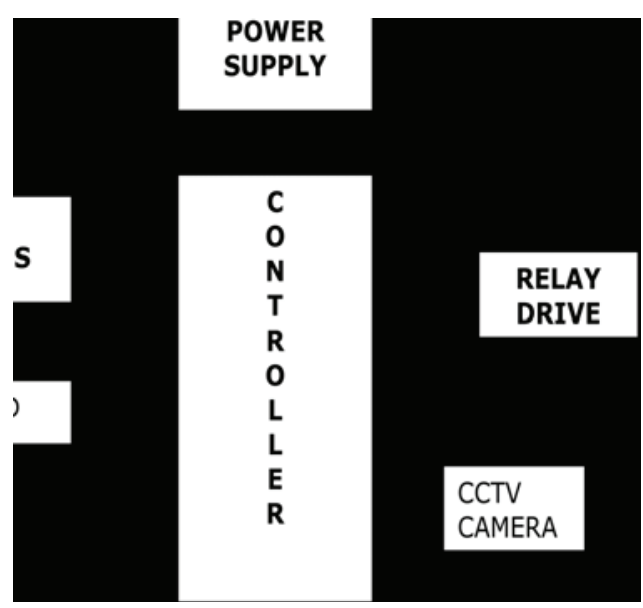

Figure 1. Diagrammatic illustration.
60 second recording period is $4.2 \mathrm{kHz}$ that gives a sound record/replay bandwidth of $20 \mathrm{~Hz}$ to $2.1 \mathrm{kHz}$. However, by changing an oscillation resistor, a sampling rate as high as $8.0 \mathrm{kHz}$ can be achieved. This shortens the total length of sound recording to 32 seconds. Total audio measuring time can be varied from 32 seconds to 60 seconds by tuning the value of a single resistor. The integrated chip can be operated in one of two modes. They are (i) serial mode and (ii) parallel mode. In serial access mode, sound can be recorded in 256 sections. In parallel access mode, sound can be recorded in 2, 4 or 8 sections. The IC can be controlled simply using push button keys. It is also possible to control the IC using external digital circuitry such as micro-controllers and computers $[2,5]$.

\section{Principle of Operation}

The APR9600 has a 28 pin DIP package. Supply voltage is between $4.5 \mathrm{~V}$ to $6.5 \mathrm{~V}$. During recording and replaying, current consumption is $25 \mathrm{~mA}$. In idle mode, the current drops to $1 \mathrm{~mA}$. The APR9600 experimental board is an assembled PCB board consisting of an APR9600 IC, an electrets microphone, support components and necessary switches to allow users to explore all.

Functions of the APR9600 chip. The oscillation resistor is chosen so that the total recording period is 60 seconds with a sampling rate of $4.2 \mathrm{kHz}$. The board measures $80 \mathrm{~mm}$ by $55 \mathrm{~mm}$.

An automatic relay is a switch which may be electrically operated control switch. Current flowing through the coil of the relay creates a magnetic field which attracts a lever and changes the switch contacts. The coil current can be ON or OFF. So relays are having two switch positions and they are double throw changeover switches. Relays allow one circuit to switch a secondary circuit which can be completely separate from the first. For example a low

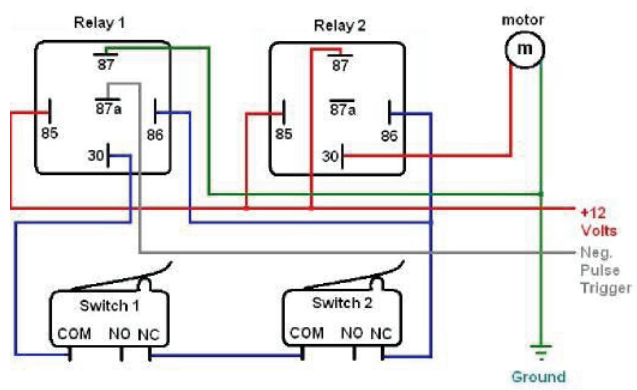

Figure 2. Circuit relay. 
voltage battery circuit can use a relay to switch a $230 \mathrm{~V}$ AC mains circuit. There is no electrical connection inside the relay between the two circuits, the linkage is magnetic and mechanical [5].

The coil of a relay passes through a relatively large current, typically $30 \mathrm{~mA}$ for a $12 \mathrm{~V}$ relay, but it can be as much as $100 \mathrm{~mA}$ for relays designed to be operated from lower voltage range. Most integrated circuit can not be provided this current and a transistor is usually used to amplify the small integrated circuit current to the larger value required for the relay coil. The maximum outputs current for the popular 555 timer integrated circuit is $200 \mathrm{~mA}$. So these devices can supply relay coils directly without amplification. Relays are usually single pole double through (SPDT) or double pole double through (DPDT) but they can have many more sets of switch contacts, for example relays with 4 sets of changeover contacts are readily available.

Most relays are designed for printed circuit board mounting but you can solder wires directly to the pins providing you take care to avoid melting the plastic case of the relay.

\section{Advantage of Relay}

Relays can be used to switch AC and DC, but the transistors can only switch DC.

Relays can switch high voltages, transistors cannot.

Relays are a better choice for switching large currents $(>5 \mathrm{~A})$.

Relays can switch many contacts at once.

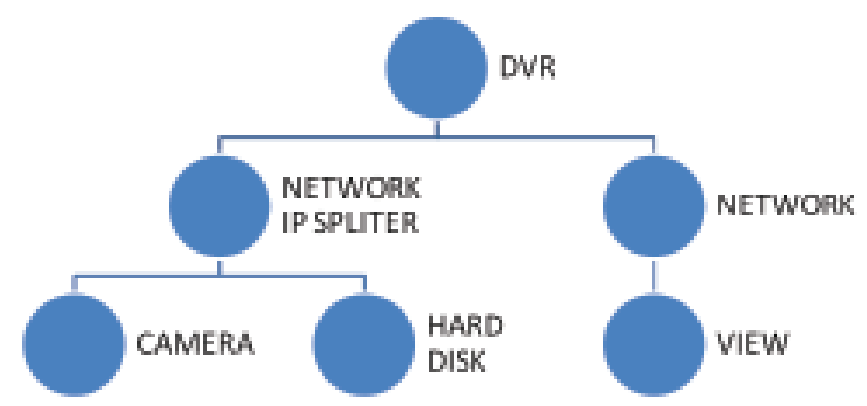

Figure 3. Sample model.

\section{Conclusion}

In real time the wheel chair is very useful for the patients especially for a handicap. We need to design micro chip memes which can be fixed into patient neck or hand. This survey concludes that the $3 \mathrm{G}$ technology provides new changes and advanced methods in telecommunication. The new $3 \mathrm{G}$ cellular phones are actually costlier when compared to traditional models. Users can do video conferencing only with other $3 \mathrm{G}$ subscribers available. There are several enhancements and features and applications possible in $3 \mathrm{G}$. The New technologies available are $4 \mathrm{G}$ and 3.5G. The main features of $4 \mathrm{G}$ are faster transfer times, better security and greater information exchanges. $4 \mathrm{G}$ can access internet and wireless services in terms of higher gigabits. The technologies can be Wi-Fi, UMTS, EDGE, or other future access technology.

\section{Acknowledgement}

We would like to thank esteemed Bharath University Research and Development, Chennai.

\section{References}

1. Kumar R, and Krogh B H (2007). A new logic programming framework for heterogeneous verification of embedded control systems, American Control Conference.

2. Embedded System Methods and Technologies-one-day workshop(2012), Los Alamitos, CA, Available From: http:// www.Schema.com

3. Korhenen J (2003). Introduction to 3G mobile communication, Technology and Engineering.

4. Ting J, and Chenglin Z (2006). Wireless sensor network technology and its application, Beijing University of posts and Telecommunication Press.

5. Lee E A (2002). Embedded software, Advances in Computers, Zelkowitz M, Ed., 56, Academic Press, London. Available from: http:// www.wikipedia.org

6. Keyence, General Catalog 2013-2014- The essential automation guide for engineers-sensors and Measurements, 7-20. Available from: http:// www.Zigbee.org 